\title{
Neoadjuvant immunotherapy facilitates resection of surgically-challenging lung squamous cell cancer
}

\author{
Ke Xü ${ }^{1 \#}$, Haitang Yang ${ }^{1 \#}$, Wenyan $\mathrm{Ma}^{2 \#}$, Liwen Fan ${ }^{1}$, Beibei Sun ${ }^{3}$, Zhexin Wang ${ }^{1}$, \\ Mohammad Faisal Al-Hurani ${ }^{4}$, Ralph A. Schmid ${ }^{5}$, Feng Yao ${ }^{1}$
}

${ }^{1}$ Department of Thoracic Surgery, Shanghai Chest Hospital, Shanghai Jiao Tong University, Shanghai, China; ${ }^{2}$ Clinical Statistical Center, Shanghai Chest Hospital, Shanghai Jiao Tong University, Shanghai, China; ${ }^{3}$ Institute for Thoracic Oncology, Shanghai Chest Hospital, Shanghai Jiao Tong University, Shanghai, China; ${ }^{4}$ Department of General and Special Surgery, Faculty of Medicine, The Hashemite University, Zarqa, Jordan; ${ }^{5}$ Division of General Thoracic Surgery, Department of BioMedical Research (DBMR), Inselspital, Bern University Hospital, University of Bern, Bern, Switzerland

Contributions: (I) Conception and design: K Xu, H Yang, F Yao; (II) Administrative support: F Yao, RA Schmid; (III) Provision of study materials or patients: K Xu, W Ma, L Fan, B Sun; (IV) Collection and assembly of data: H Yang, K Xu, MF Al-Hurani; (V) Data analysis and interpretation: K Xu, W Ma, Z Wang; (VI) Manuscript writing: All authors; (VII) Final approval of manuscript: All authors.

"These authors contributed equally to this work.

Correspondence to: Haitang Yang; Feng Yao. Department of Thoracic Surgery, Shanghai Chest Hospital, Shanghai Jiao Tong University, West Huai 241, Shanghai 200030, China. Email: xyxm8889@163.com; yaofeng@shsmu.edu.cn.

Background: Locally-advanced lung squamous cell carcinoma represents a special subset that is challenging to resect completely with surgery alone. Immunotherapy has achieved great success in treating late-stage lung cancer. However, whether neoadjuvant immunotherapy can facilitate resection of initially locally-advanced and surgically-difficult locally-advanced lung squamous cell carcinoma remains to be investigated.

Methods: We retrospectively collected clinical records of locally-advanced lung squamous cell carcinoma patients who received neoadjuvant immunotherapy followed by surgery between 2018 and 2020 at a large academic thoracic cancer center.

Results: A total of 23 patients (22 males, 1 female) with locally-advanced locally-advanced lung squamous cell carcinoma were included, initially clinically staged at IIIA (16, 69.6\%), IIIB ( $\mathrm{n}=4,17.4 \%)$, IIB ( $\mathrm{n}=2$, $8.7 \%)$ and IIIC ( $\mathrm{n}=1,4.3 \%)$. The median interval between final treatment to surgery was 36 days (range, 25-93 days), without treatment-related delay in surgery. The neoadjuvant treatment resulted in a high rate of radical resection $(n=20,87.0 \%)$. The final histopathological examination demonstrated $6(26.1 \%)$ cases with pathological complete response and 8 (34.8\%) with pathological major response. Comparing with the computed tomography scan-based response, we observed a very low consistency (weighted kappa $=0.122$, $\mathrm{P}=0.315$ ) between the computed tomography scan-based and final pathological evaluation. The median follow-up time was 510 days (range, 217-920 days). At the end of the follow-up, 1 patient died.

Conclusions: Our findings showed the clinical promise of neoadjuvant immunotherapy plus surgery for locally-advanced lung squamous cell carcinoma. Computed tomography scan displays a poor role in assessing the resectability after neoadjuvant immunotherapy.

Keywords: Surgery; neoadjuvant immunotherapy; lung squamous cell carcinoma (LSqCC); advanced stage; pathological response

Submitted Jul 21, 2021. Accepted for publication Sep 23, 2021.

doi: $10.21037 /$ jtd-21-1195

View this article at: https://dx.doi.org/10.21037/jtd-21-1195

(C) Journal of Thoracic Disease. All rights reserved. 


\section{Introduction}

Lung squamous cell carcinoma (LSqCC) represents a major subtype of non-small cell lung cancer (NSCLC) with limited treatment options due to the absence of actionable driver mutations. Most cases of LSqCC are centrallylocated and diagnosed at locally-advanced stages, making it challenging to resect completely.

For the treatment of cancer patients, neoadjuvant therapy is defined as the administration of therapeutic agents before surgical resection. There are several advantages of neoadjuvant therapy, e.g., a direct pathological evaluation of treatment efficacy, increasing the resectability by downstaging the lung tumors, improving the rate of lungsparing surgery, and increasing distant and local disease control. Meanwhile, there are also some concerns, such as delaying the timing of curative treatment, increasing treatment toxicity, and confounding accurate pathological staging. Furthermore, previous evidence demonstrated heterogeneous clinical outcomes of surgery after neoadjuvant chemotherapy or chemo-radiotherapy in locally-advanced NSCLC (1-3), leading to little advances in neoadjuvant therapy worldwide (4). As such, for locallyadvanced NSCLC, the role of neoadjuvant therapy remains controversial.

Immune checkpoint inhibitors (ICIs), either alone or in combination with platinum doublet-based chemotherapy, have shown survival benefits for patients with metastatic NSCLC $(5,6)$. Besides, maintenance treatment with programmed death-ligand 1 (PD-L1) inhibitors prolonged survival in locally-advanced NSCLC patients who had received concurrent chemoradiation when compared with placebo control $(7,8)$. In the setting of LSqCC specifically, ICIs-based treatment strategy has been clinically approved [CheckMate 017 (9), Keynote-40 (5), RATIONALE 307 (10)]. Based on these promising results, recent attention has focused on exploring ICIs as a neoadjuvant treatment strategy, followed by surgery, in locally-advanced NSCLC, which showed early clinical promise (11-13). However, whether neoadjuvant can facilitate the radical resection in the case of surgically challenging LSqCC remains to be investigated.

In this retrospective study, we analyzed the clinical data of surgically challenging locally-advanced LSqCC patients who had received surgery after neoadjuvant immunotherapy or chemo-immunotherapy. We aim to evaluate whether neoadjuvant immunotherapy or chemo-immunotherapy enables a high rate of radical resection with promising clinical outcomes for these patients. In addition, the secondary endpoint is to assess the accuracy of computed tomography (CT) scans to predict pathological responses. We presented the following article in accordance with the STROBE reporting checklist (available at https://dx.doi. org/10.21037/jtd-21-1195).

\section{Methods}

\section{Patients}

We retrospectively reviewed the clinical records of NSCLC patients who underwent surgery between January 2018 and December 2020 at Shanghai Chest Hospital. Inclusion criteria: (I) patients who had a pathological diagnosis of LSqCC; (II) patients whose tumors were challenging to resect completely evaluated by a multidisciplinary board of lung cancer; (III) patients who received immunotherapy or chemo-immunotherapy before surgery. Surgicallychallenging lung cancer cases were defined as (I) NSCLC tumors of any size which have spread to the contralateral lymph nodes, or have invaded other essential structures or organs in the chest such as the large vessels (e.g., superior vena cava, aorta), heart, trachea, esophagus or vertebral body; (II) involvement of multiple mediastinal lymph nodes (N2); (III) the presence of malignant pleural or pericardial effusion; (IV) patients who were potentially intolerable to extensive resections due to the limited cardiopulmonary function reserve and/or comorbidities.

Clinical data (demographic data, clinical and pathological characteristics, therapeutic regimens, and clinical outcomes) of patients who received neoadjuvant immunotherapy or chemo-immunotherapy were retrieved for further analysis. The study was conducted in accordance with the Declaration of Helsinki (as revised in 2013). This study was approved by the institutional review board [\#KS(Y)21039]. All patients had signed informed consent for inclusion of their clinical data and specimens in our Lung Biobank and use in research projects, according to the recommendation of the ethics committee of Shanghai Chest Hospital.

\section{Preoperative examinations}

All patients underwent routinely preoperative examinations to assess the surgical resectability and safety, including brain magnetic resonance imaging (MRI), contrastenhanced chest CT scan or positron emission tomography (PET)/CT scan, bronchoscopy, abdominal CT or 
ultrasonography examination, and/or whole-body bone scan. The pathological confirmation was based on tumor biopsies, PET-CT or endobronchial ultrasound-guided transbronchial needle aspiration (EBUS-TBNA) was used to evaluate mediastinal nodal status. All patients who underwent surgery had an Eastern Cooperative Oncology Group performance status of $0-1$, normal organ function, and sufficient lung function reserve for resection.

\section{Neoadjuvant immunotherapy}

Lung tumors harboring actionable mutations, such as epidermal growth factor receptor (EGFR), echinoderm microtubule-associated protein-like 4-anaplastic lymphoma kinase fusion oncogene (EML4-ALK), repressor of silencing 1 (ROS1), or mesenchymal epithelial transition factor (MET), were routinely examined and excluded from immunotherapy. Neoadjuvant therapy-related information, such as agents, courses, doses, and duration of final neoadjuvant treatment to surgery, was collected. For patients receiving ICI alone, one of the approved ICIs was administrated: (I) pembrolizumab [Keytruda (Merck), anti-programmed cell death protein 1 (PD-1), at a dose of $200 \mathrm{mg}$ intravenously every 3 weeks]; (II) nivolumab [Opdivo (Bristol-Myers Squibb), anti-PD-1, at a dose of $240 \mathrm{mg}$ intravenously every 2 weeks]; (III) sintilimab (antiPD-1; jointly developed by Innovent Biologics and Eli Lilly, at a dose of $200 \mathrm{mg}$ intravenously every 3 weeks); (IV) or ipilimumab [Yervoy (Bristol-Myers Squibb), anti-cytotoxic T-lymphocyte-associated protein 4 (CTLA-4), at a dose of $3 \mathrm{mg}$ per kilogram, intravenously every 3 weeks]. For patients receiving chemo-immunotherapy, single ICI plus platinum doublets were given. Patients received the first dose of immunotherapy concurrently with chemotherapy, and the subsequent doses were administered every 2 or 3 weeks (according to the corresponding indications) in a total of $2-5$ cycles, and chemotherapy was given every 3 weeks in a total of 2-3 cycles. For patients receiving ICI(s) alone, PDL1 expression with tumor proportion score (TPS) $\geq 50 \%$ was routinely applied, while chemo-immunotherapy was given irrespective of the PD-L1 TPS expression.

\section{Operation}

Posterolateral thoracotomy (open) or video-assisted thoracic surgery (VATS) with systemic lymphadenectomy was performed based on the patient's conditions, patients' informed consent, and surgeons' preference. The operative approach, the extent of resection, operative time, hospital length of stay, postoperative morbidity, and other details related to the operative experience, were recorded in detail. Clinical and pathological staging of patients were evaluated according to The American Joint Committee on Cancer (AJCC) Lung Cancer Staging (8th edition) (14). Surgical complications were documented according to the criteria defined by the Society of Thoracic Surgeons and the European Society of Thoracic Surgeons general thoracic surgery databases (15). In addition, we also use the Clavien-Dindo classification (16) as the complement for the traditional classification methods.

All patients received postoperative adjuvant immunotherapy or chemotherapy ( \pm radiation) after being discussed by the multidisciplinary tumor board.

\section{Treatment response assessments}

After completion of neoadjuvant treatment, PET/CT or CT scan was performed to evaluate therapeutic response and resectability, preferably 2 weeks after the last dose of therapy. The response was assessed based on the Response Evaluation Criteria in Solid Tumors (RECIST) (version 1.1) (17). Later, patients who did not have disease progress underwent surgery in 5-6 weeks. Pathological complete response (pCR) was defined as the absence of viable tumor cells (ypT0NOM0) in the surgical resection specimen; pathological major response $(\mathrm{pMR})$ was defined as $10 \%$ or less viable tumor cells in the surgical resection specimen; pathological partial response (pPR) was defined as more than $10 \%$ viable tumor cells in the surgical resection specimen. The pathological response was graded according to the Junker criteria and assessed in all resected specimens (18).

\section{Smoking and PD-L1 subgroups}

Cumulative smoking exposure was determined in terms of pack-years by multiplying the number of years smoked with the average number of packs per day (19). Based on packyears of smoking, subjects were classified as never smokers (0.0 pack-years), light smokers (0.1-20.0 pack-years), moderate smokers (20.1-40.0 pack-years), and heavy smokers (>40 pack-years) (20).

Tumoral PD-L1 expression was scored by a senior pathologist according to the PD-L1 tumor proportions score before neoadjuvant immunotherapy, and samples were divided into three subgroups: PD-L1-high ( $\geq 50 \%)$, -moderate (1-50\%), and -low $(<1 \%)$. 


\section{Immunobistochemistry}

Serial $5 \mu \mathrm{m}$ tissue sections from biopsies and the matched resected primary tumors were deparaffinized, rehydrated, pretreated for antigen retrieval, and stained with hematoxylin and eosin and subjected to immunohistochemical staining following a standard protocol $(19,21)$. The following primary antibodies were used: anti-human PD-L1 (DaLo, monoclonal mouse anti-human, clone 22C3; Copenhagen, Denmark).

\section{Follow-up}

The first follow-up visit was scheduled 4 weeks after discharge. Then adjuvant therapies were typically started at 1 month postoperatively. Later, follow-up visits were scheduled every 3 months with chest CT scans, brain MRI, abdominal sonography or CT, and serum tumor markers. Other examinations were performed according to the oncologists when necessary. Follow-up information was obtained from patients by phone calls or clinic re-visit records.

Recurrence-free survival (RFS) was defined as the interval between the day of pathological diagnosis and the date of detected tumor relapse by any cause or the last follow-up date. Overall survival (OS) was defined as the interval between the day of pathological diagnosis and the date of death by any cause or the last follow-up date (April 15, 2021).

\section{Statistical analysis}

Normally distributed continuous variables were presented as mean \pm standard deviation (SD), otherwise as median and range (21). Categorical variables were shown as numbers and percentages. Survival analysis was performed using "survminer" and "survival" R packages. Kappa Test was performed for agreement between CT-based evaluation and final histopathological examination of treatment response using the function 'kappa2' from the package 'irr' in R. Data summary and statistical analysis were performed using $\mathrm{R}$ software (version 3.6.3) (22). $\mathrm{P}<0.05$ was considered significant.

\section{Results}

\section{Clinical characteristics}

A total of 23 surgically-challenging LSqCC cases (22 males and 1 female) who received neoadjuvant immunotherapy or chemo-immunotherapy were included in this study. The clinical characteristics of patients and tumors included in this study cohort were shown in Table 1 .

\section{Neoadjuvant immunotberapy or chemo-immunotberapy}

None of the patients received preoperative radiotherapy. The detailed treatment regimens involving immunotherapy or chemo-immunotherapy were summarized in Table 2. Patients received a median of 3 doses of ICIs before resection (range, 2-5 doses). Six patients (26.1\%) received neoadjuvant immunotherapy alone, including single $(\mathrm{n}=5)$ and double $(n=1)$ ICIs, and 17 patients $(73.9 \%)$ were managed with neoadjuvant chemo-immunotherapy.

All neoadjuvant treatment-related adverse events $(n=9,39.1 \%)$ were manageable. Immunotherapy/chemoimmunotherapy related adverse events occurred in 9 patients, including pneumonia of grade II-III $(n=3)$, slight-to-moderate liver dysfunction $(n=3)$, slight-tomoderate bone marrow suppression $(n=2)$, and nausea/ vomiting $(n=1)$. The median interval between the last cycle of therapy and surgery was 36 days (range, 25-93 days), without treatment-related delay in surgery.

After neoadjuvant immunotherapy or chemoimmunotherapy, a post-treatment CT scan showed that 19 patients $(82.6 \%)$ had a partial response, and 4 patients $(17.4 \%)$ had stable disease, and there was no evidence of disease progression.

\section{Surgical resection and postoperative course}

The most common resection type was standard lobectomy $(13,56.5 \%)$, followed by sleeve lobectomy (6, 26.1\%), pneumonectomy $(3,13.0 \%)$, and bilobectomy (1, 4.3\%). Minimally invasive approaches were performed in 14 $(60.9 \%)$ patients. One patient $(4.3 \%)$ required conversion to thoracotomy due to the adhesions. Finally, among the 23 patients undergoing surgery, 20 (87.0\%) achieved complete resections and $3(13.0 \%)$ had positive bronchial margins. The positive surgical margin was due to the limited lung function reserve, which might be detrimental to the patients if a more extensive resection was performed.

The median hospital postoperative stay was 7 days (range, 2-29 days). One or more postoperative manageable complications occurred in 5 out of the 23 patients, of which 4 of grade I-II (minor complications) and 1 of grade III-IV (major complications), according to the ClavienDindo classification. To be specifically, the most common 
Table 1 Clinicopathological characteristics

\begin{tabular}{|c|c|c|}
\hline Characteristics & Level & No. (total $\mathrm{N}=23$ ) \\
\hline \multirow[t]{2}{*}{ Gender (\%) } & Female & $1(4.3)$ \\
\hline & Male & $22(95.7)$ \\
\hline Age (years), mean (SD) & & $61.0(8.0)$ \\
\hline \multirow[t]{4}{*}{ Smoking history (\%) } & Heavy & $7(30.4)$ \\
\hline & Light & $3(13.0)$ \\
\hline & Moderate & $8(34.8)$ \\
\hline & Never & $5(21.7)$ \\
\hline \multirow[t]{2}{*}{ Serum tumor markers (\%) } & High & $18(78.3)$ \\
\hline & Normal & $5(21.7)$ \\
\hline \multirow[t]{3}{*}{ Diagnostic approach (\%) } & EBUS & $2(8.7)$ \\
\hline & PNLB & $8(34.8)$ \\
\hline & TBB & $13(56.5)$ \\
\hline \multirow[t]{4}{*}{ Location (\%) } & LL & $1(4.3)$ \\
\hline & LU & $8(34.8)$ \\
\hline & $R L$ & $4(17.4)$ \\
\hline & $R U$ & $10(43.5)$ \\
\hline Histology (\%) & LUSC & $23(100.0)$ \\
\hline \multirow[t]{3}{*}{ Surgical approach (\%) } & Open & $8(34.8)$ \\
\hline & VATS & $14(60.9)$ \\
\hline & VATS to open & $1(4.3)$ \\
\hline \multirow[t]{4}{*}{ PD-L1 expression (\%) } & High & $7(30.4)$ \\
\hline & Low & $2(8.7)$ \\
\hline & Moderate & $1(4.3)$ \\
\hline & Unknown & $13(56.5)$ \\
\hline \multirow[t]{2}{*}{ Neoadjuvant treatment (\%) } & $\mathrm{ICl}$ alone & $6(26.1)$ \\
\hline & $\begin{array}{l}\text { ICI plus } \\
\text { chemotherapy }\end{array}$ & $17(73.9)$ \\
\hline \multirow[t]{4}{*}{ Clinical stage (\%) } & IIB & $2(8.7)$ \\
\hline & IIIA & $16(69.6)$ \\
\hline & IIIB & $4(17.4)$ \\
\hline & IIIC & $1(4.3)$ \\
\hline \multirow[t]{4}{*}{ Surgical resection (\%) } & Lobectomy & $13(56.5)$ \\
\hline & Bilobectomy & $1(4.3)$ \\
\hline & Sleeve & $6(26.1)$ \\
\hline & Pneumonectomy & $3(13.0)$ \\
\hline
\end{tabular}

Table 1 (continued)
Table 1 (continued)

\begin{tabular}{llc}
\hline Characteristics & Level & No. (total N=23) \\
\hline Resection margin (\%) & Negative & $20(87.0)$ \\
& Positive & $3(13.0)$ \\
Pathological response (\%) & pCR & $6(26.1)$ \\
& pMR & $8(34.8)$ \\
& pPR & $9(39.1)$ \\
\hline
\end{tabular}

EBUS, endobronchial ultrasound; PNLB, percutaneous needle lung biopsy; TBB, transbronchial biopsy; LL, left lower; LU, left upper; RL, right lower; RU, right upper; LUSC, lung squamous cell carcinoma; VATS, video-assisted thoracoscopic surgery; PD-L1, programmed death-ligand 1; ICl, immune checkpoint inhibitors; pCR, pathological complete response; pMR, pathological major response; pPR, pathological partial response.

Table 2 Neoadjuvant regimens

\begin{tabular}{ll}
\hline Immunotherapy regimens & No. \\
\hline ICl(s) alone & 3 \\
Nivolumab & 2 \\
Pembrolizumab & 1 \\
Nivolumab + ipilimumab & \\
Single ICl plus platinum doublets & 5 \\
Pembrolizumab + paclitaxel plus platinum & 4 \\
Pembrolizumab + pemetrexed plus platinum & 3 \\
Nivolumab + gemcitabine plus platinum & 2 \\
Nivolumab + docetaxel plus platinum & 1 \\
Pembrolizumab + gemcitabine plus platinum & 1 \\
Nivolumab + paclitaxel plus platinum & 1 \\
Nivolumab + pemetrexed plus platinum
\end{tabular}

ICls, immune checkpoint inhibitors.

complication was prolonged air leak $(n=3)$, followed by pneumonia $(n=1)$, chylothorax $(n=1)$. There was one surgery-related death of a patient with a bronchopleural fistula (BPF) on postoperative day 29. We hypothesized that the extensive dissection required for a proximal tumor and preoperative chemotherapy may be the risk factors for BPF. This patient suffered life-threatening complications secondary to the BPF including pneumonia and respiratory failure which attributed to the poor outcome. Overall, the 30 -day mortality rate was $4.3 \%$. 


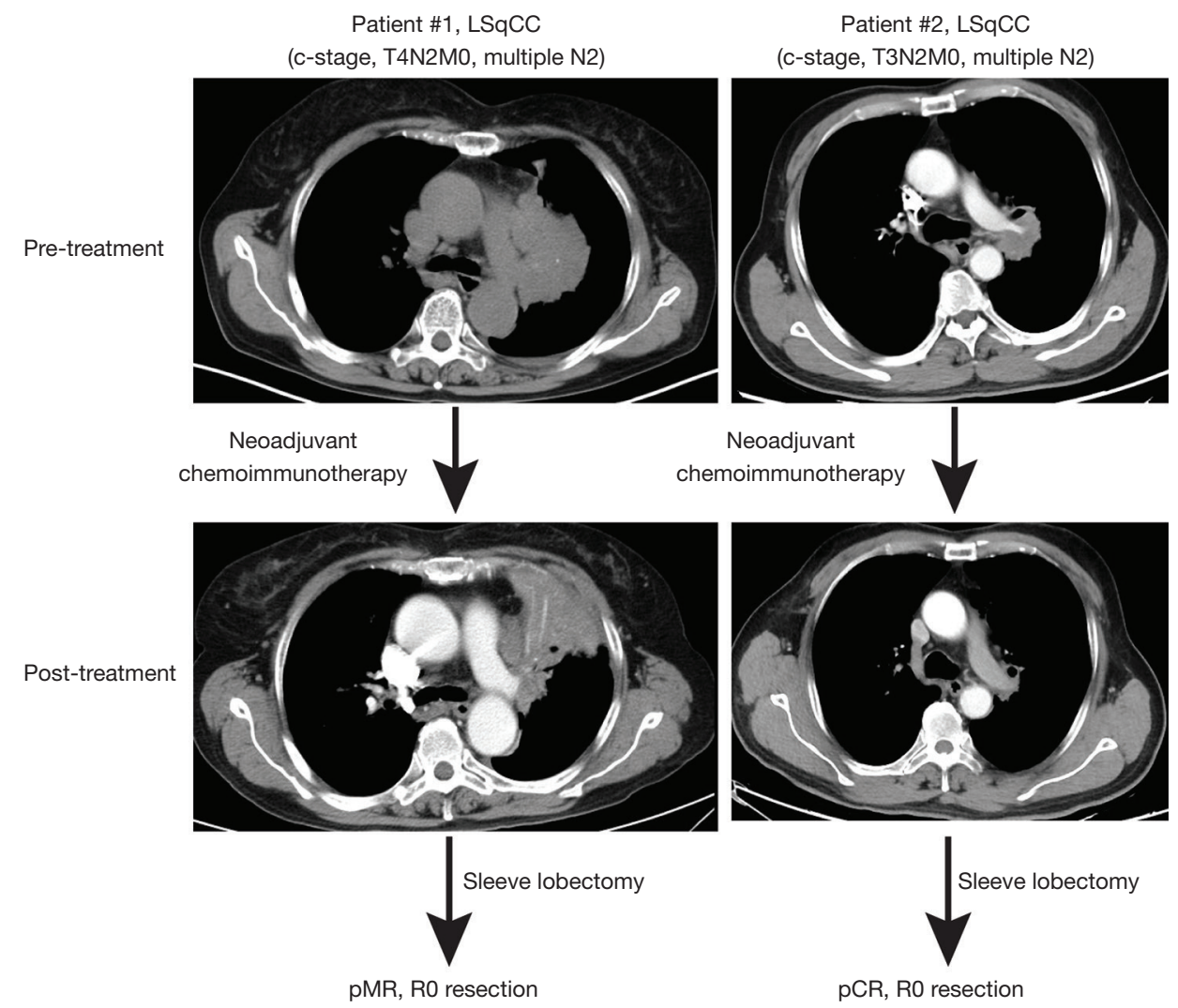

Figure 1 Representative computed tomography (CT) scans showing two cases of locally-advanced and surgically challenging lung squamous cell carcinoma (LSqCC) pre-and post-neoadjuvant chemo-immunotherapy. Patient \#1 had a pathological major response (pMR) to immunotherapy; patient \#2 had a pathological complete response (pCR) to immunotherapy in the final examinations. After neoadjuvant chemo-immunotherapy, both cases got a radical resection (R0).

\section{Pathological response}

Finally, the postoperative pathological examination demonstrated $6(26.1 \%)$ cases with pCR, $8(34.8 \%)$ with $\mathrm{pMR}$, and $9(39.1 \%)$ with pPR (Table 1; Figure 1). Comparing with the CT scan-based response, we observed a very low consistency (weighted kappa $=-0.125, \mathrm{P}=0.315$ ) between the CT scan-based and final pathological evaluation (Table 3), suggesting a poor role of CT scan in evaluating the real treatment response to ICIs. Additionally, we observed two cases with complete response in the primary tumor bed, but tumor cells in the involved lymph nodes still exist (ypT0N2M0), which might represent a resistant subset clone to immunotherapy, potentially leading to tumor relapse.

\section{Survival}

The median follow-up time was 510 days (range, 217-920 days). At the end of follow-up, 5 (21.7\%; 3 with $\mathrm{pPR}$ and 2 with $\mathrm{pCR}$ in response to neoadjuvant immunotherapy) patients had tumor relapse and one patient (pPR in response to neoadjuvant immunotherapy) died of tumor recurrence. Two-year RFS and OS were $84.40 \%$ and 94.1\%, respectively (Figure 2).

A graphic summary of this study was shown in Figure 3.

\section{Discussion}

Over the past decade, there has been little advance in the neoadjuvant treatment of locally-advanced LSqCC, in 
Table 3 Inconsistency between CT-scan-based and pathological evaluation of treatment response

\begin{tabular}{lcccc}
\hline Response & CT-scan, $\mathrm{n}(\%)$ & Pathology, $\mathrm{n}(\%)$ & P value & Weighted kappa \\
\hline SD & $4(17.4)$ & 0 & 0.315 & 0.122 \\
PR & $19(82.6)$ & $17^{\#}(73.9)$ & & \\
CR & 0 & $6(26.1)$ & & \\
\hline
\end{tabular}

", including 8 cases with major response. CT, computed tomography; SD, stable disease; PR, partial response; CR, complete response.

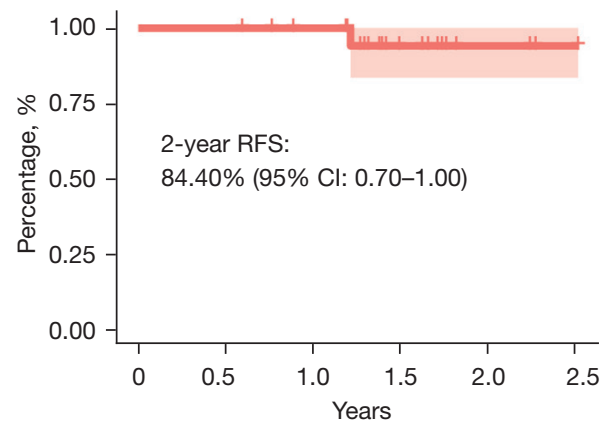

Number at risk

$\begin{array}{llllll}22 & 22 & 19 & 9 & 3 & 1\end{array}$

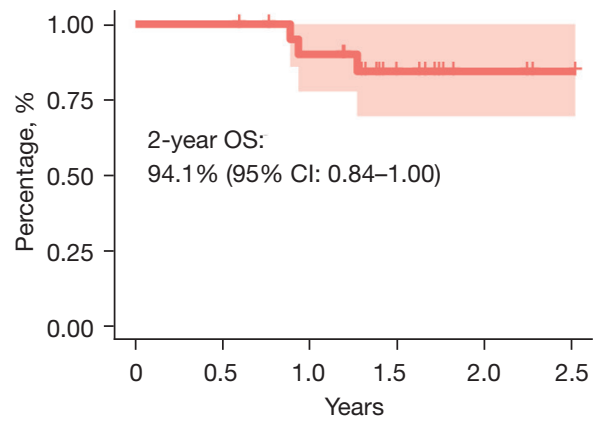

Number at risk

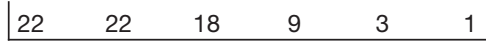

Figure 2 RFS and OS of lung cancer patients in this study cohort. Here, one patient who died of surgery-related complications was excluded. RFS, recurrence-free survival; OS, overall survival; CI, confidence interval.

Combined immunotherapy and surgery as a new multimodal strategy for locally-advanced lung squamous cell cancer

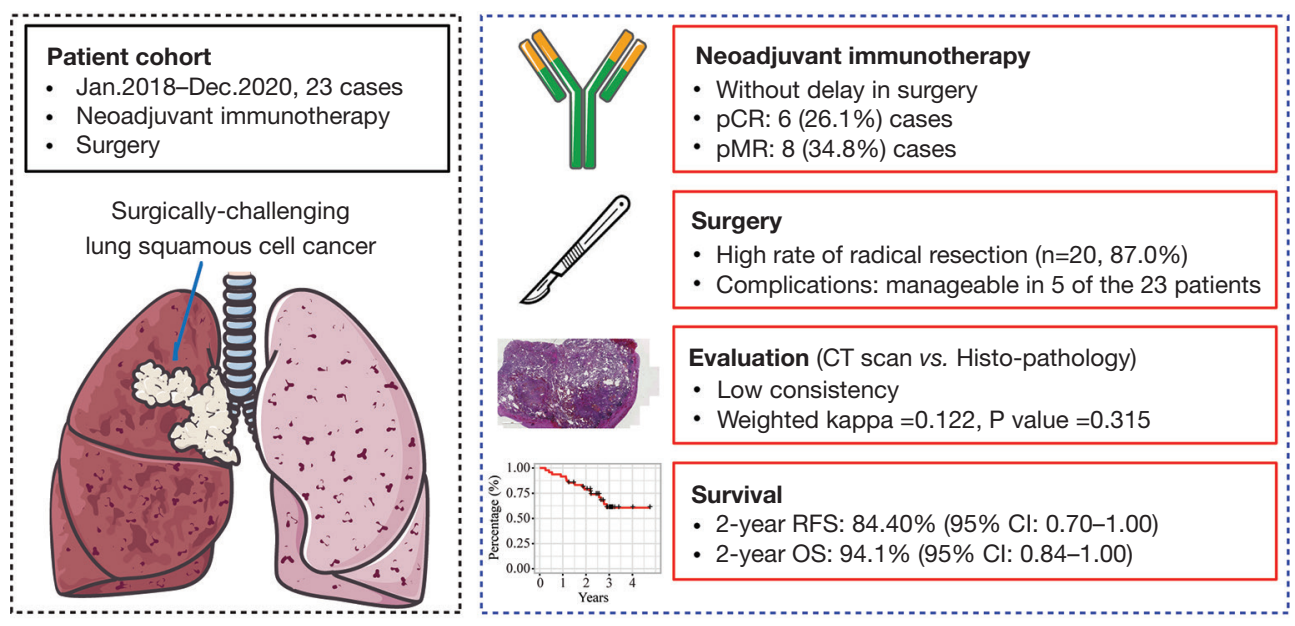

1. Neoadjuvant immunotherapy facilitates resection of surgically-demanding lung squamous cell cancer

2. CT scan-based evaluation cannot well reflect therapeutic response

Figure 3 A graphic summary of this study. pCR, pathological complete response; pMR, pathological major response; CT, computed tomography. 
which a minority of tumors could be resected by surgical removal alone. Conventional neoadjuvant chemotherapy and/or radiotherapy is routinely recommended to this subset of patients, although there was no substantial improvement of patient survival, with rates of distant metastases still frequent and pCRs observed only in a minority of patients (23-25). Thus, it is urgently needed to explore novel treatment strategies to reduce the tumor burden and improve the pCR rate, and prolong the survival of $\mathrm{LSqCC}$ patients whose tumors are potentially unresectable. Emerging data from clinical trials have demonstrated that immunotherapy substantially improves the survival of patients with late-stage NSCLC, including advanced $\operatorname{LSqCC}(5,6,26)$, and has also clinical promise in early-stage NSCLC (27). However, in the real-world setting, studies specifically investigating neoadjuvant immunotherapy followed by surgery for locally-advanced and surgically difficult LSqCC are lacking.

\section{The rationale of neoadjuvant immunotherapy for $L S q C C$}

Neoantigen burden is regarded as a fundamental determinant of response to immunotherapy (28). Accordingly, tumor mutational burden (TMB), which is associated with neoantigen burden, has been approved as a PD-L1-independent biomarker predicting the therapeutic responses to ICIs (29).

Locally-advanced $\mathrm{LSqCC}$ is often detected as a large tumor that relates to high antigen burden, providing a certain rationale for immunotherapy in this setting. LSqCC is typically associated with heavy exposure to tobacco. In our study cohort, $78.3 \%$ of patients have a smoking history. Interestingly, recent evidence shows that smoking history is a biomarker predicting the therapeutic responses to ICIs in NSCLC (30-32). Gainor et al. retrospectively analyzed a cohort of NSCLC patients treated with ICIs, there is a dramatic difference in the overall response rate in heavy smokers, compared with that in light or never smokers (20.6\% vs. 4.2\%) (33). Mechanistically, smoking could lead to high TMB and/or PD-L1 (34-36), which, at least partially, explains the association between heavy smoking history and high response rate to ICIs treatment. Further studies are warranted to investigate the mechanistic links of heavy smoking history with the response to ICIs treatment.

\section{Chemo-immunotherapy versus chemo-free immunotherapy}

There are potential synergies between immunotherapy and chemotherapy $(37,38)$, which was confirmed by several phase III clinical trials demonstrating that in late-stage NSCLC patients, the addition of immunotherapy to chemotherapy could dramatically prolong patient survival with manageable safety and tolerability $(5,6,39)$. Despite this, heterogeneous outcomes were also observed (40).

Clinical evidence has revealed that in the neoadjuvant setting (41), ICIs doublets are better than single ICI. Recently, FDA approved a chemo-free combination of nivolumab plus ipilimumab as a first-line treatment for patients with metastatic NSCLC that expresses PD-L1 $(\geq 1 \%)$ (26). More recently, a phase II trial demonstrated for the first time promising clinical outcomes of chemofree neoadjuvant nivolumab plus ipilimumab followed by surgery in patients with stage I-III operable NSCLC (41). Currently, immunotherapy, either as double ICIs alone or in combination with chemotherapy, has demonstrated superior efficacy to chemotherapy only in the frontline setting. However, evidence on the choice of chemo-immunotherapy or chemo-free immunotherapy as the front-line treatment of NSCLC is still lacking, and a variety of factors, such as cost-effectiveness, toxicity, and efficacy, need to be weighed.

\section{The role of surgery}

Our data demonstrated neo-adjuvant immunotherapy or chemo-immunotherapy resulted in a high radical resection in the surgically difficult LSqCC cases. Whether surgery is necessary and can provide a survival benefit remains to be defined due to the lack of long-term follow-up. Our clinical experience informed us that CT scan-based approach could not truly reflect the treatment response (Figure 1; Table 3), and there is also a lack of actionable biomarkers to accurately predict the treatment response to neoadjuvant ICIs. Moreover, in our cohort, we observed two cases with complete response in the primary tumor bed, but we still observed tumor cells in the matched lymph nodes, which might represent a resistant subset clone to immunotherapy. Therefore, surgery is still a necessity, which requires further studies.

Besides, CT scan appears to be less helpful for resectability after neoadjuvant immunotherapy. In our study, all cases have a partial response, including 14 cases $(60.9 \%)$ with a high degree of pathological response, in the final histological examination, which, however, was not reflected on CT images.

\section{Limitations}

This study has some limitations, such as the inherent bias 
of a retrospective study with small sample size. Also, the patients were highly selected. The definition of surgicallychallenging patients is heterogeneous. Besides, the patients included in this study were also heterogeneous regarding the given neoadjuvant treatment. For example, the number of cell cycles of neoadjuvant immunotherapy is different between individuals which might affect the subsequent surgical resections. Validation with independent cohorts and prospective studies is warranted.

\section{Conclusions}

In this study, we provided a piece of real-world evidence showing promising clinical outcomes of neoadjuvant immunotherapy for patients with locally-advanced NSCLC and surgically demanding LSqCC. Our data support combined neoadjuvant immunotherapy and surgery as a new multimodal strategy for the management of this special surgically difficult subset of LSqCC.

\section{Acknowledgments}

Funding: This research was supported by the Science and Technology Development project of Shanghai Chest Hospital and Shanghai Municipality (to Y Feng).

\section{Footnote}

Reporting Checklist: The authors have completed the STROBE reporting checklist. Available at https://dx.doi. org/10.21037/jtd-21-1195

Data Sharing Statement: Available at https://dx.doi. org/10.21037/jtd-21-1195

Peer Review File: Available at https://dx.doi.org/10.21037/ jtd-21-1195

Conflicts of Interest: All authors have completed the ICMJE uniform disclosure form (available at https://dx.doi. org/10.21037/jtd-21-1195). The authors have no conflicts of interest to declare.

Ethical Statement: The authors are accountable for all aspects of the work in ensuring that questions related to the accuracy or integrity of any part of the work are appropriately investigated and resolved. The study was conducted in accordance with the Declaration of Helsinki (as revised in 2013). The participants gave informed consent before taking part. The study was approved by ethics board of Shanghai Chest Hospital [\#KS(Y)21039].

Open Access Statement: This is an Open Access article distributed in accordance with the Creative Commons Attribution-NonCommercial-NoDerivs 4.0 International License (CC BY-NC-ND 4.0), which permits the noncommercial replication and distribution of the article with the strict proviso that no changes or edits are made and the original work is properly cited (including links to both the formal publication through the relevant DOI and the license). See: https://creativecommons.org/licenses/by-nc-nd/4.0/.

\section{References}

1. Pu CY, Yendamuri S. Neoadjuvant versus adjuvant chemotherapy for resectable non-small cell lung cancer debate revisited. J Thorac Dis 2019;11:5646-8.

2. Uprety D, Mandrekar SJ, Wigle D, et al. Neoadjuvant Immunotherapy for NSCLC: Current Concepts and Future Approaches. J Thorac Oncol 2020;15:1281-97.

3. Yang H, Yao F, Zhao Y, et al. Clinical outcomes of surgery after induction treatment in patients with pathologically proven N2-positive stage III non-small cell lung cancer. J Thorac Dis 2015;7:1616-23.

4. Brascia D, De Iaco G, Schiavone M, et al. Resectable IIIA-N2 Non-Small-Cell Lung Cancer (NSCLC): In Search for the Proper Treatment. Cancers (Basel) 2020;12:2050.

5. Paz-Ares L, Luft A, Vicente D, et al. Pembrolizumab plus Chemotherapy for Squamous Non-Small-Cell Lung Cancer. N Engl J Med 2018;379:2040-51.

6. Gandhi L, Rodríguez-Abreu D, Gadgeel S, et al. Pembrolizumab plus Chemotherapy in Metastatic NonSmall-Cell Lung Cancer. N Engl J Med 2018;378:2078-92.

7. Faivre-Finn C, Vicente D, Kurata T, et al. Four-Year Survival With Durvalumab After Chemoradiotherapy in Stage III NSCLC-an Update From the PACIFIC Trial. J Thorac Oncol 2021;16:860-7.

8. Antonia SJ, Villegas A, Daniel D, et al. Overall Survival with Durvalumab after Chemoradiotherapy in Stage III NSCLC. N Engl J Med 2018;379:2342-50.

9. Brahmer J, Reckamp KL, Baas P, et al. Nivolumab versus Docetaxel in Advanced Squamous-Cell Non-Small-Cell Lung Cancer. N Engl J Med 2015;373:123-35.

10. Wang J, Lu S, Yu X, et al. Tislelizumab Plus Chemotherapy vs Chemotherapy Alone as First-line 
Treatment for Advanced Squamous Non-Small-Cell Lung Cancer: A Phase 3 Randomized Clinical Trial. JAMA Oncol 2021;7:709-17.

11. Reuss JE, Anagnostou V, Cottrell TR, et al. Neoadjuvant nivolumab plus ipilimumab in resectable non-small cell lung cancer. J Immunother Cancer 2020;8:e001282.

12. Jiang L, Huang J, Jiang S, et al. The surgical perspective in neoadjuvant immunotherapy for resectable nonsmall cell lung cancer. Cancer Immunol Immunother 2021;70:2313-21.

13. Ren S, Wang C, Shen J, et al. Neoadjuvant immunotherapy with resectable non-small cell lung cancer: recent advances and future challenges. J Thorac Dis 2020;12:1615-20.

14. Detterbeck FC, Boffa DJ, Kim AW, et al. The Eighth Edition Lung Cancer Stage Classification. Chest 2017;151:193-203.

15. Fernandez FG, Falcoz PE, Kozower BD, et al. The Society of Thoracic Surgeons and the European Society of Thoracic Surgeons general thoracic surgery databases: joint standardization of variable definitions and terminology. Ann Thorac Surg 2015;99:368-76.

16. Clavien PA, Barkun J, de Oliveira ML, et al. The ClavienDindo classification of surgical complications: five-year experience. Ann Surg 2009;250:187-96.

17. Schwartz LH, Litière $\mathrm{S}$, de Vries $\mathrm{E}$, et al. RECIST 1.1-Update and clarification: From the RECIST committee. Eur J Cancer 2016;62:132-7.

18. Forde PM, Chaft JE, Smith KN, et al. Neoadjuvant PD-1 Blockade in Resectable Lung Cancer. N Engl J Med 2018;378:1976-86.

19. Yang H, Liang SQ, Xu D, et al. HSP90/AXL/eIF4Eregulated unfolded protein response as an acquired vulnerability in drug-resistant KRAS-mutant lung cancer. Oncogenesis 2019;8:45.

20. Lee YH, Shin MH, Kweon SS, et al. Cumulative smoking exposure, duration of smoking cessation, and peripheral arterial disease in middle-aged and older Korean men. BMC Public Health 2011;11:94.

21. Yang H, Xu J, Yao F, et al. Analysis of unexpected small cell lung cancer following surgery as the primary treatment. J Cancer Res Clin Oncol 2018;144:2441-7.

22. Yang H, Al-Hurani MF, Xu J, et al. pN1 but not pN0/N2 predicts survival benefits of prophylactic cranial irradiation in small-cell lung cancer patients after surgery. Ann Transl Med 2021;9:562.

23. Dickhoff C, Senan S, Schneiders FL, et al. Ipilimumab plus nivolumab and chemoradiotherapy followed by surgery in patients with resectable and borderline resectable T3-
4N0-1 non-small cell lung cancer: the INCREASE trial. BMC Cancer 2020;20:764.

24. Eberhardt WE, Pöttgen C, Gauler TC, et al. Phase III Study of Surgery Versus Definitive Concurrent Chemoradiotherapy Boost in Patients With Resectable Stage IIIA(N2) and Selected IIIB Non-Small-Cell Lung Cancer After Induction Chemotherapy and Concurrent Chemoradiotherapy (ESPATUE). J Clin Oncol 2015;33:4194-201.

25. Albain KS, Swann RS, Rusch VW, et al. Radiotherapy plus chemotherapy with or without surgical resection for stage III non-small-cell lung cancer: a phase III randomised controlled trial. Lancet 2009;374:379-86.

26. Hellmann MD, Paz-Ares L, Bernabe Caro R, et al. Nivolumab plus Ipilimumab in Advanced Non-Small-Cell Lung Cancer. N Engl J Med 2019;381:2020-31.

27. Mielgo-Rubio X, Calvo V, Luna J, et al. Immunotherapy Moves to the Early-Stage Setting in Non-Small Cell Lung Cancer: Emerging Evidence and the Role of Biomarkers. Cancers (Basel) 2020;12:3459.

28. Jiang T, Shi T, Zhang H, et al. Tumor neoantigens: from basic research to clinical applications. J Hematol Oncol 2019;12:93.

29. Marabelle A, Fakih M, Lopez J, et al. Association of tumour mutational burden with outcomes in patients with advanced solid tumours treated with pembrolizumab: prospective biomarker analysis of the multicohort, openlabel, phase 2 KEYNOTE-158 study. Lancet Oncol 2020;21:1353-65.

30. Li JJN, Karim K, Sung M, et al. Tobacco exposure and immunotherapy response in PD-L1 positive lung cancer patients. Lung Cancer 2020;150:159-63.

31. Norum J, Nieder C. Tobacco smoking and cessation and PD-L1 inhibitors in non-small cell lung cancer (NSCLC): a review of the literature. ESMO Open 2018;3:e000406.

32. Ng TL, Liu Y, Dimou A, et al. Predictive value of oncogenic driver subtype, programmed death-1 ligand (PD-L1) score, and smoking status on the efficacy of PD-1/PD-L1 inhibitors in patients with oncogene-driven non-small cell lung cancer. Cancer 2019;125:1038-49.

33. Gainor JF, Shaw AT, Sequist LV, et al. EGFR Mutations and ALK Rearrangements Are Associated with Low Response Rates to PD-1 Pathway Blockade in Non-Small Cell Lung Cancer: A Retrospective Analysis. Clin Cancer Res 2016;22:4585-93.

34. Doukas SG, Vageli DP, Lazopoulos G, et al. The Effect of NNK, A Tobacco Smoke Carcinogen, on the miRNA and Mismatch DNA Repair Expression Profiles in Lung 
and Head and Neck Squamous Cancer Cells. Cells 2020;9:1031.

35. Stabile LP, Kumar V, Gaither-Davis A, et al. Syngeneic tobacco carcinogen-induced mouse lung adenocarcinoma model exhibits PD-L1 expression and high tumor mutational burden. JCI Insight 2021;6:145307.

36. Wang X, Ricciuti B, Nguyen T, et al. Association between Smoking History and Tumor Mutation Burden in Advanced Non-Small Cell Lung Cancer. Cancer Res 2021;81:2566-73.

37. Emens LA, Middleton G. The interplay of immunotherapy and chemotherapy: harnessing potential synergies. Cancer Immunol Res 2015;3:436-43.

38. He X, Du Y, Wang Z, et al. Upfront dose-reduced chemotherapy synergizes with immunotherapy to optimize chemoimmunotherapy in squamous cell lung carcinoma. J Immunother Cancer 2020;8:e000807.

39. Gadgeel S, Rodríguez-Abreu D, Speranza G, et al. Updated Analysis From KEYNOTE-189: Pembrolizumab or Placebo Plus Pemetrexed and Platinum for Previously Untreated Metastatic Nonsquamous Non-Small-Cell Lung Cancer. J Clin Oncol 2020;38:1505-17.

40. Govindan R, Szczesna A, Ahn MJ, et al. Phase III Trial of Ipilimumab Combined With Paclitaxel and Carboplatin in Advanced Squamous Non-Small-Cell Lung Cancer. J Clin Oncol 2017;35:3449-57.

41. Cascone T, William WN Jr, Weissferdt A, et al. Neoadjuvant nivolumab or nivolumab plus ipilimumab in operable non-small cell lung cancer: the phase 2 randomized NEOSTAR trial. Nat Med 2021;27:504-14.

Cite this article as: $\mathrm{Xu} \mathrm{K}$, Yang $\mathrm{H}, \mathrm{Ma} W$, Fan L, Sun B, Wang Z, Al-Hurani MF, Schmid RA, Yao F. Neoadjuvant immunotherapy facilitates resection of surgically-challenging lung squamous cell cancer. J Thorac Dis 2021;13(12):6816-6826. doi: $10.21037 /$ jtd-21-1195 